Supporting Information

\title{
$\mathrm{Cl}^{-}$-templated Assembly of Novel Peanut-like $\mathrm{Ln}_{40} \mathrm{Ni}_{44}$ Heterometallic Clusters Exhibiting Large Magnetocaloric Effect
}

Ning-Fang Li, ${ }^{\dagger, \#}$ Qing-Fang Lin, ${ }^{\dagger,},{ }^{*}$ Xi-Ming Luo, ${ }^{\dagger}$ Jia-Peng Cao, ${ }^{\dagger}$ and Yan Xu ${ }^{*}, \dot{*}$, ${ }^{\dagger}$ College of Chemical Engineering, State Key Laboratory of Materials-Oriented Chemical Engineering, Nanjing Tech University, Nanjing 210009, P. R. China †Department of Chemistry, Bengbu Medical College, Bengbu 233030, PR China 


\section{Physical Measurements}

Elemental analyses (C, H and N) were performed on a Perkin-Elmer 2400 elemental analyzer.

Powder X-ray diffraction (PXRD) patterns were recorded on a Bruker D8X diffractometer equipped with monochromatized $\mathrm{Cu}-\mathrm{K}_{\alpha}(\lambda=1.5418 \AA)$ radiation at room temperature. Data were collected in the range of 5-50 ${ }^{\circ}$.

IR spectra of compounds 1-3 were recorded on a Nicolet Impact 410 FTIR spectrometer with pressed $\mathrm{KBr}$ pellets, from 4000 to $400 \mathrm{~cm}^{-1}$.

Thermogravimetric (TG) measurements were carried out on a Diamond thermogravimetric analyzer in a flowing $\mathrm{N}_{2}$ atmosphere from 25 to $800{ }^{\circ} \mathrm{C}$, with a heating rate of $10^{\circ} \mathrm{C} \cdot \mathrm{min}^{-1}$.

The SEM images and energy dispersive spectrometer (EDS) were determined by using a Hitachi S-4800 scanning electron microscope at an accelerating voltage of 20 $\mathrm{kV}$.

The variable temperature magnetic susceptibility data were measured over the temperature range of $1.8-300 \mathrm{~K}$ and the isothermal magnetization measurements were carried out between 0 and $7 \mathrm{~T}$, using a Quantum Design MPMS-XL 7 SQUID magnetometer. The experimental magnetic susceptibility data for all compounds were corrected for diamagnetic contributions estimated using Pascal's constant and of the sample holder by a previous calibration. 
Coordination modes of metal ions $\left(\mathrm{Gd}^{3+}, \mathrm{Eu}^{3+}, \mathrm{Ni}^{2+}\right)$

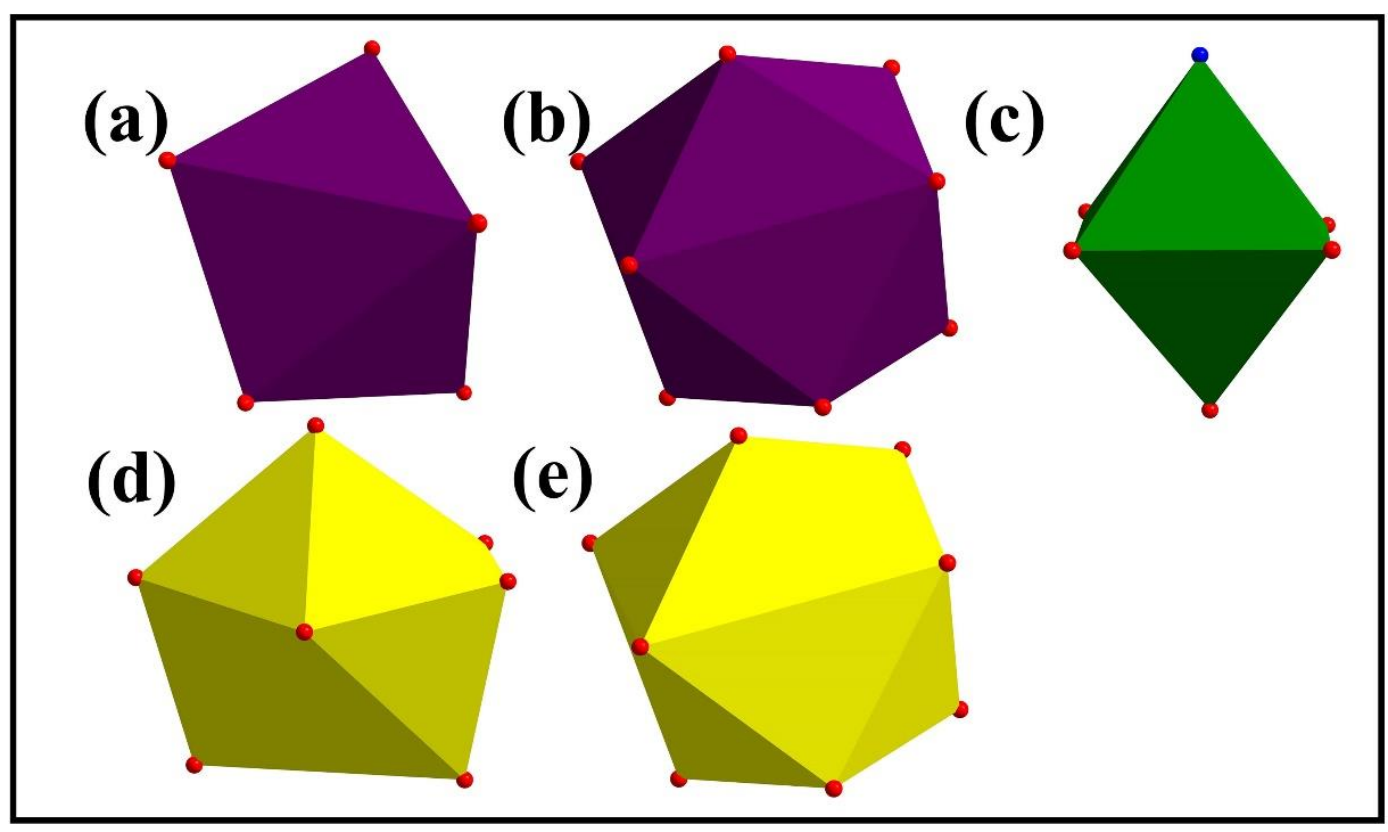

Figure S1. The coordination mode of $\mathrm{Eu}^{3+}(\mathrm{a}-\mathrm{b}), \mathrm{Ni}^{2+}(\mathrm{c}), \mathrm{Gd}^{3+}(\mathrm{d}-\mathrm{e})$.

\section{Coordination modes of ligands}

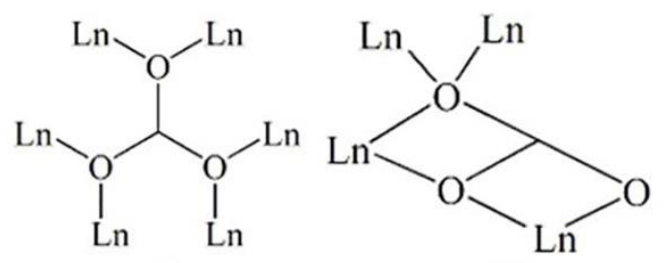

(a)

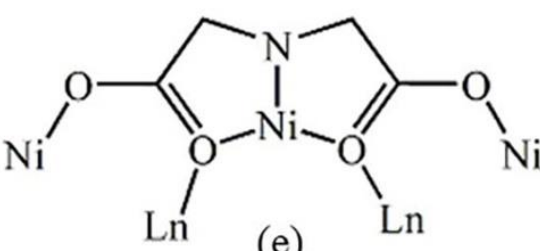

(e)

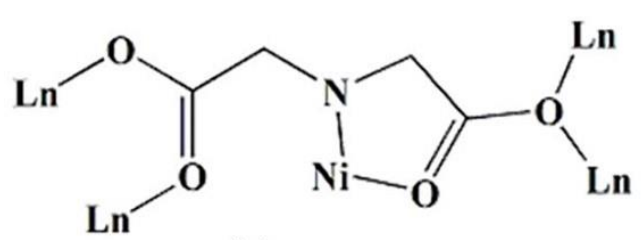

(g)

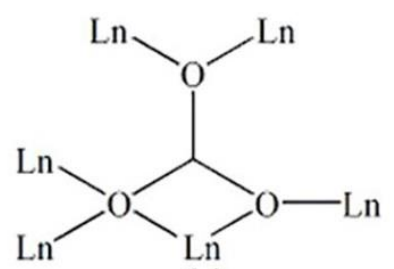

(c)

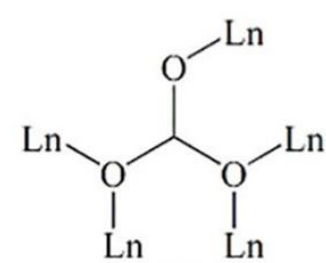

(d)

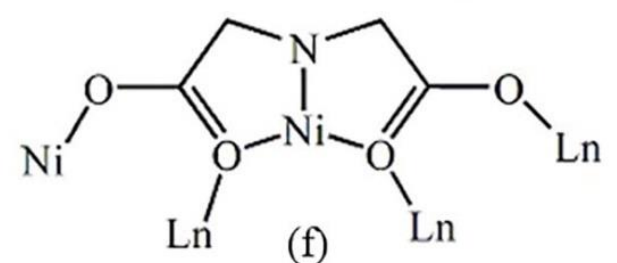<smiles>C1OC2O[AlH]OC2O[Al]1</smiles>

(h)<smiles>CC1O[Al]O1</smiles>

(i) 
Figure S2. The coordination mode of $\mathrm{CO}_{3}{ }^{2-}(\mathrm{a}-\mathrm{d}), \mathrm{IDA}(\mathrm{e}-\mathrm{g})$, oxalic acid (h) and acetate (i).

\section{Structural views}

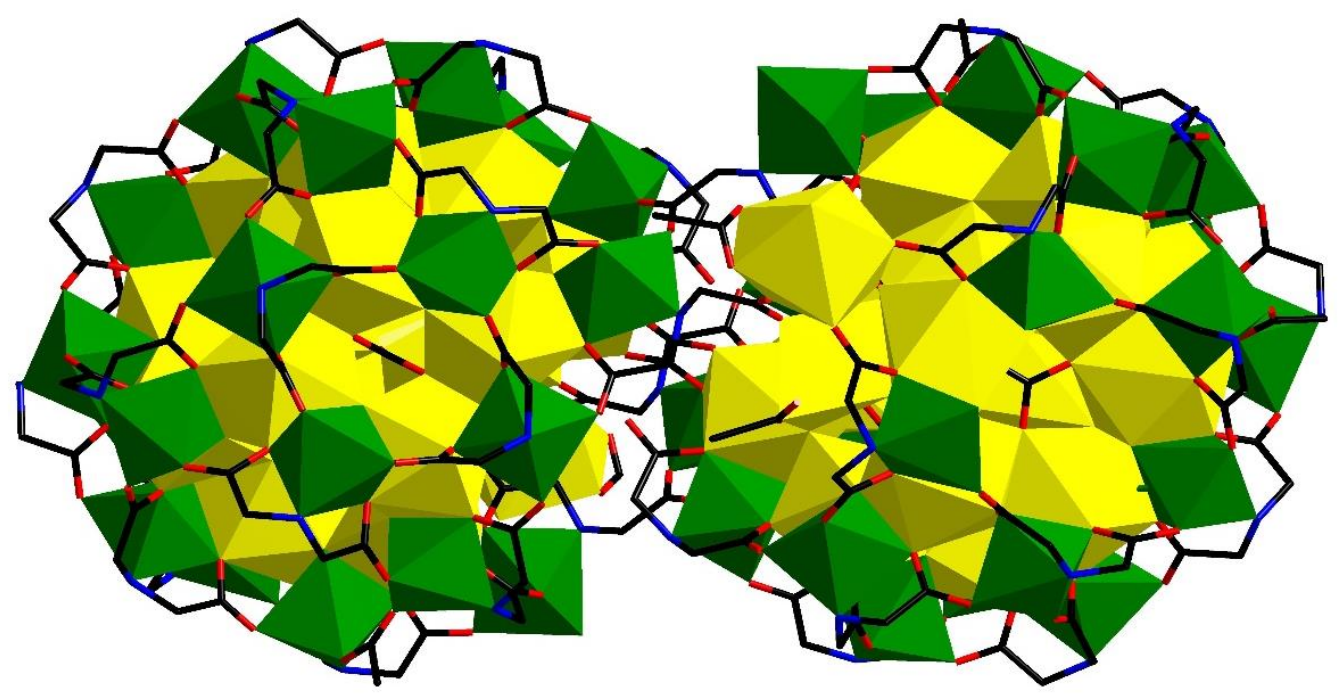

Figure S3. (a) The polyhedron view of the cationic cluster of 2. (The $\mathrm{H}$ atoms and free $\mathrm{Cl}^{-}$and water were deleted for clearness; green: $\mathrm{Ni}^{2+}$, yellow: $\mathrm{Gd}^{3+}$, black: $\mathrm{C}$, blue: $\mathrm{N}$, red: $\mathrm{O})$.

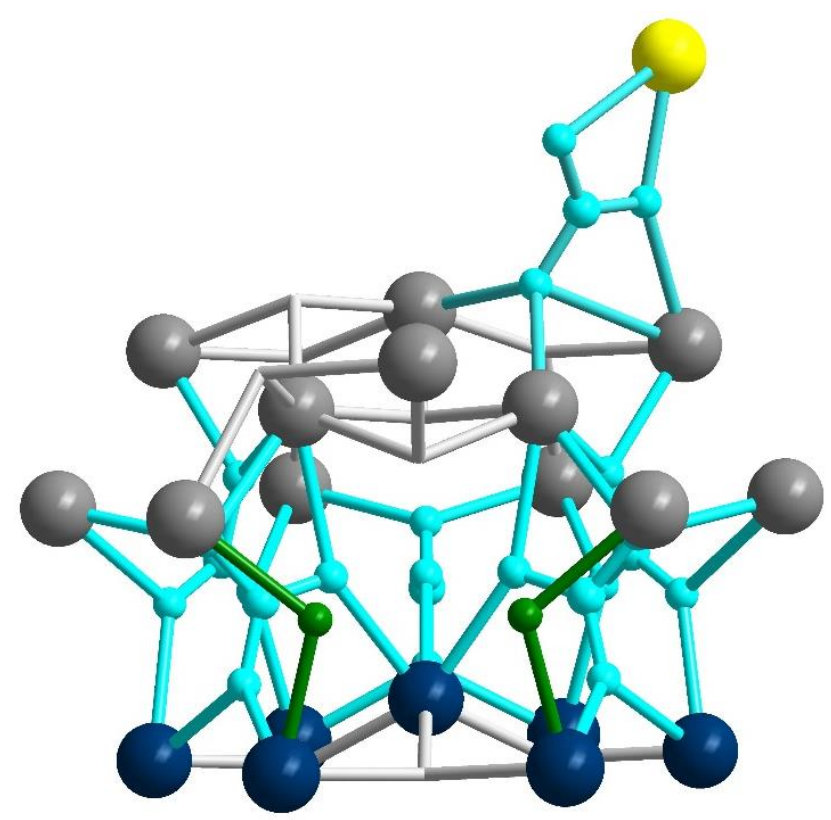

Figure S4. A ball-and-stick view of the unit of $\mathbf{E \mathbf { u } _ { 2 0 }}$. (green: $\mu_{2}-\mathrm{OH}$ group, turquoise: $\mathrm{CO}_{3}{ }^{2-}$ group). 


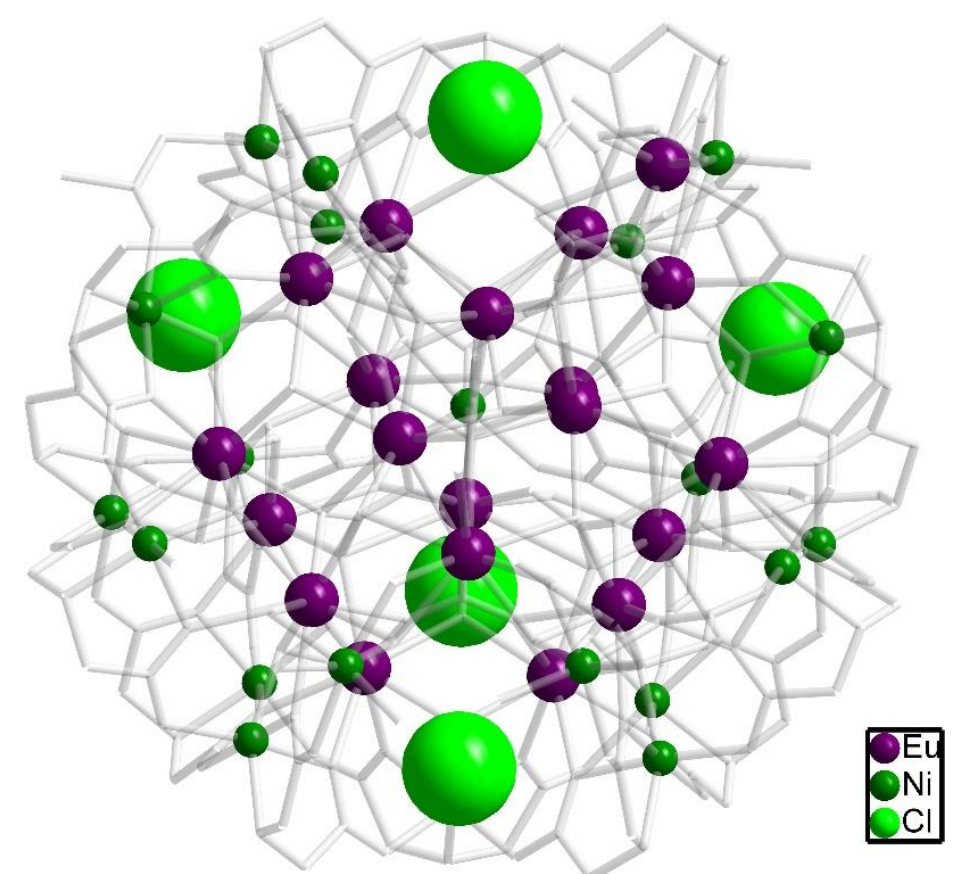

Figure S5. (a) A view of the $\mathbf{E u}_{20} \mathbf{N i}_{22}$ (yellow: $\mathrm{Eu}^{3+}$, blue: $\mathrm{Ni}^{2+}$, bright green: $\mathrm{Cl}^{-}$).

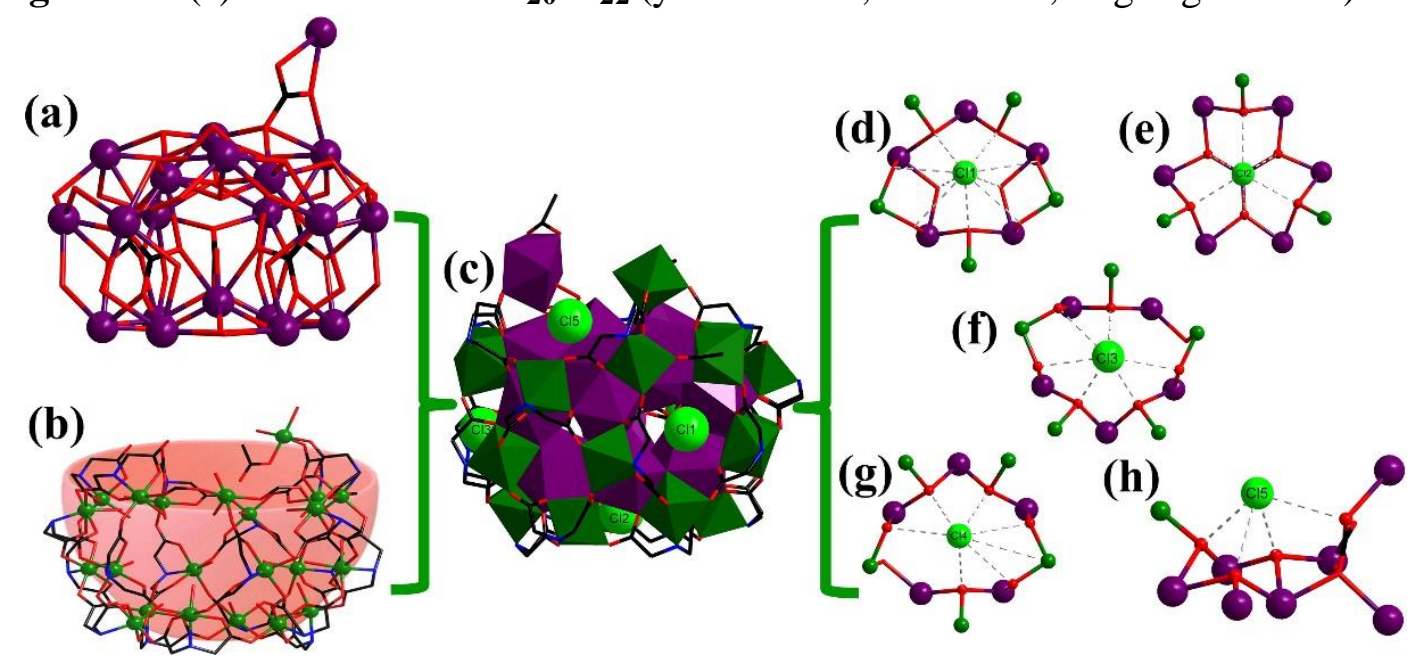

Figure S6. (a) A ball-and-stick view of the unit of $\mathbf{E} \mathbf{u}_{20}$, (b) A view of ball-like $\mathbf{N i} \mathbf{i}_{22}$ (purple: $\mathrm{Eu}^{3+}$, blue: $\mathrm{Ni}^{2+}$ ), (c) A view of the $\mathbf{E u}_{20} \mathbf{N i}_{22}$, (d) A view of $\left[\mathrm{Eu}_{5} \mathrm{Ni}_{5}\left(\mu_{3}-\mathrm{OH}\right)_{5} \mathrm{Cl}\right]^{19+}$ unit, (e) A view of $\left[\mathrm{Eu}_{6} \mathrm{Ni}_{5}\left(\mu_{3}-\mathrm{OH}\right)_{6} \mathrm{Cl}\right]^{21+}$ unit, (f) A view of $\left[\mathrm{Eu}_{4} \mathrm{Ni}_{5} \mathrm{Cl}\right]^{21+}$ unit, (g) A view of $\left[\mathrm{Eu}_{5} \mathrm{Ni}_{5}\left(\mu_{3}-\mathrm{OH}\right)_{3} \mathrm{Cl}\right]^{21+}$ unit, (h) A view of $\left[\mathrm{Eu}_{7} \mathrm{Ni}\left(\mathrm{CO}_{3}\right)\left(\mu_{3}-\mathrm{OH}\right)_{3} \mathrm{Cl}\right]^{16+}$ unit. 
(a)

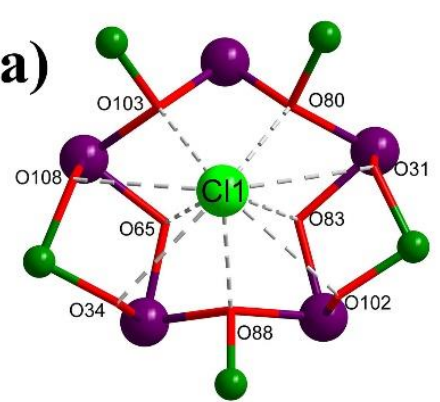

(d)

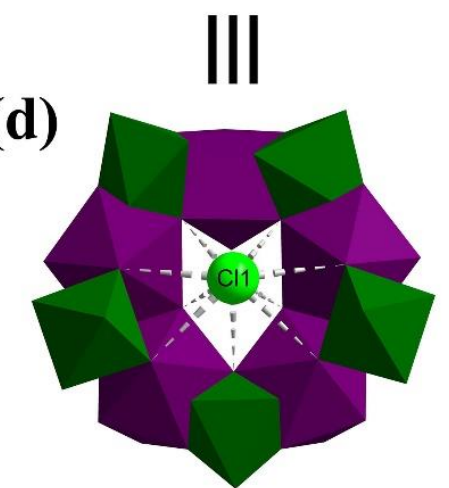

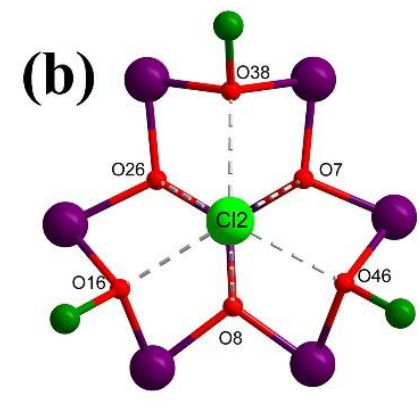

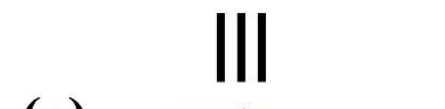

(e)

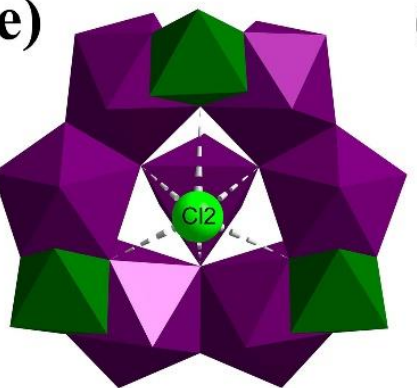

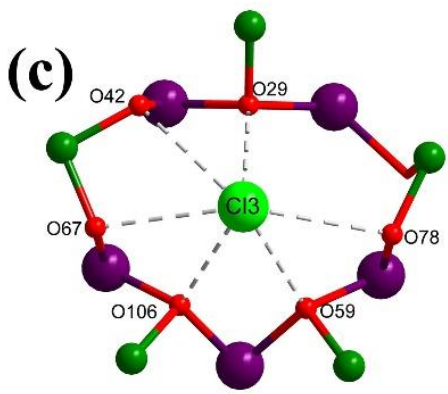

(f)

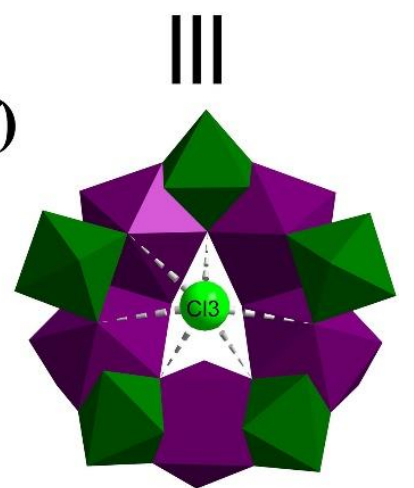

Figure S7. (a), (d) A view of $\left[\mathrm{Eu}_{5} \mathrm{Ni}_{5}\left(\mu_{3}-\mathrm{OH}\right)_{5} \mathrm{Cl}\right]^{19+}$ unit; (b), (e) A view of $\left[\mathrm{Eu}_{6} \mathrm{Ni}_{5}\left(\mu_{3}-\mathrm{OH}\right)_{6} \mathrm{Cl}\right]^{21+}$ unit; (c), (f) $\mathrm{A}$ view of $\left[\mathrm{Eu}_{4} \mathrm{Ni}_{5} \mathrm{Cl}\right]^{21+}$ unit.

(a)

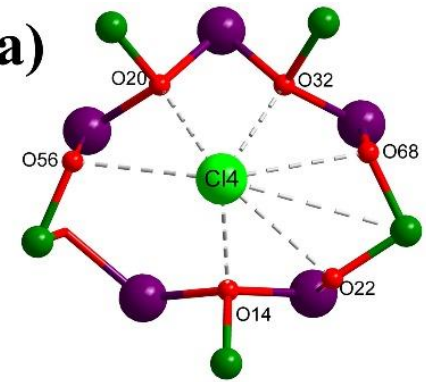

(c)

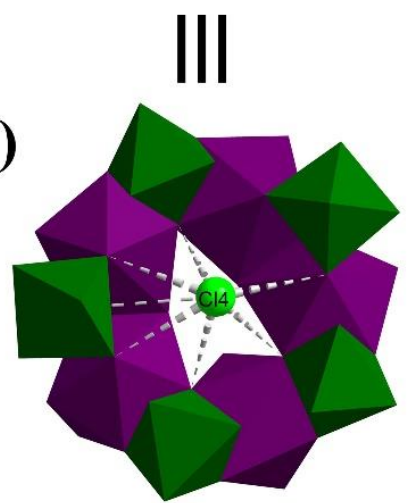

(b)

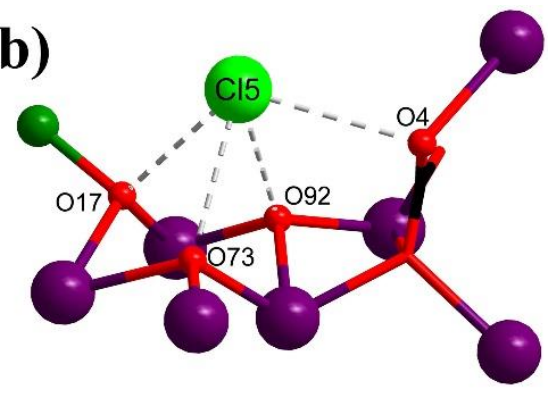

(d)

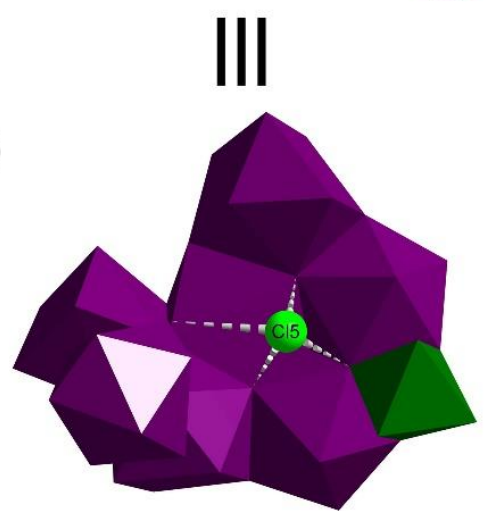

Figure S8. (a), (c) A view of $\left[\mathrm{Eu}_{5} \mathrm{Ni}_{5}\left(\mu_{3}-\mathrm{OH}\right)_{3} \mathrm{Cl}\right]^{21+}$ unit; (b), (d) A view of $\left[\mathrm{Eu}_{7} \mathrm{Ni}\left(\mathrm{CO}_{3}\right)\left(\mu_{3}-\mathrm{OH}\right)_{3} \mathrm{Cl}\right]^{16+}$ unit. 
XRD

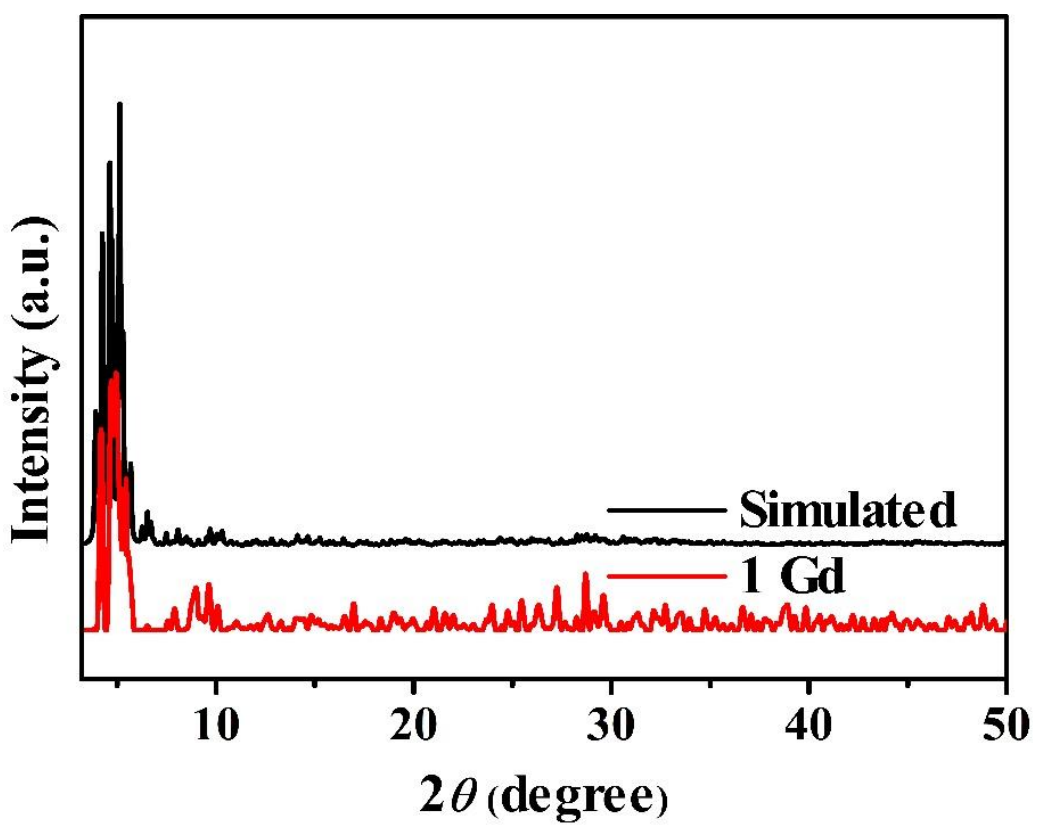

Figure S9. PXRD patterns of all compounds 1.

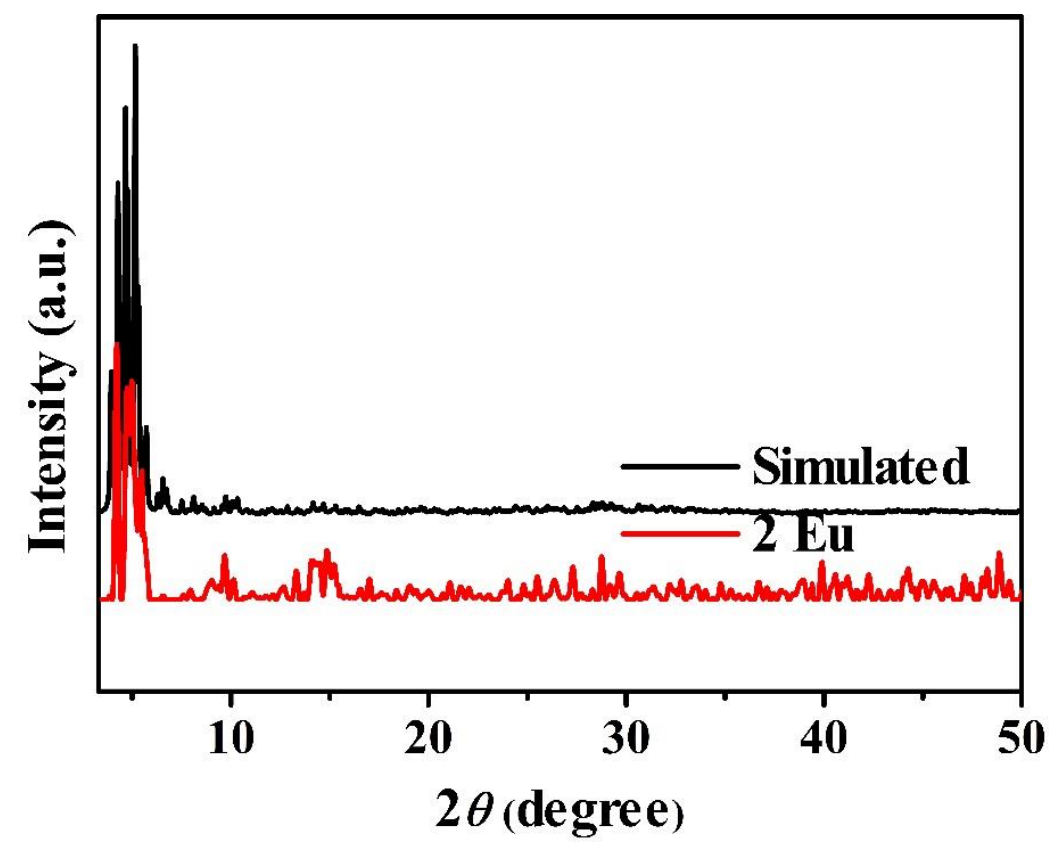

Figure S10. PXRD patterns of all compounds 2 . 
IR

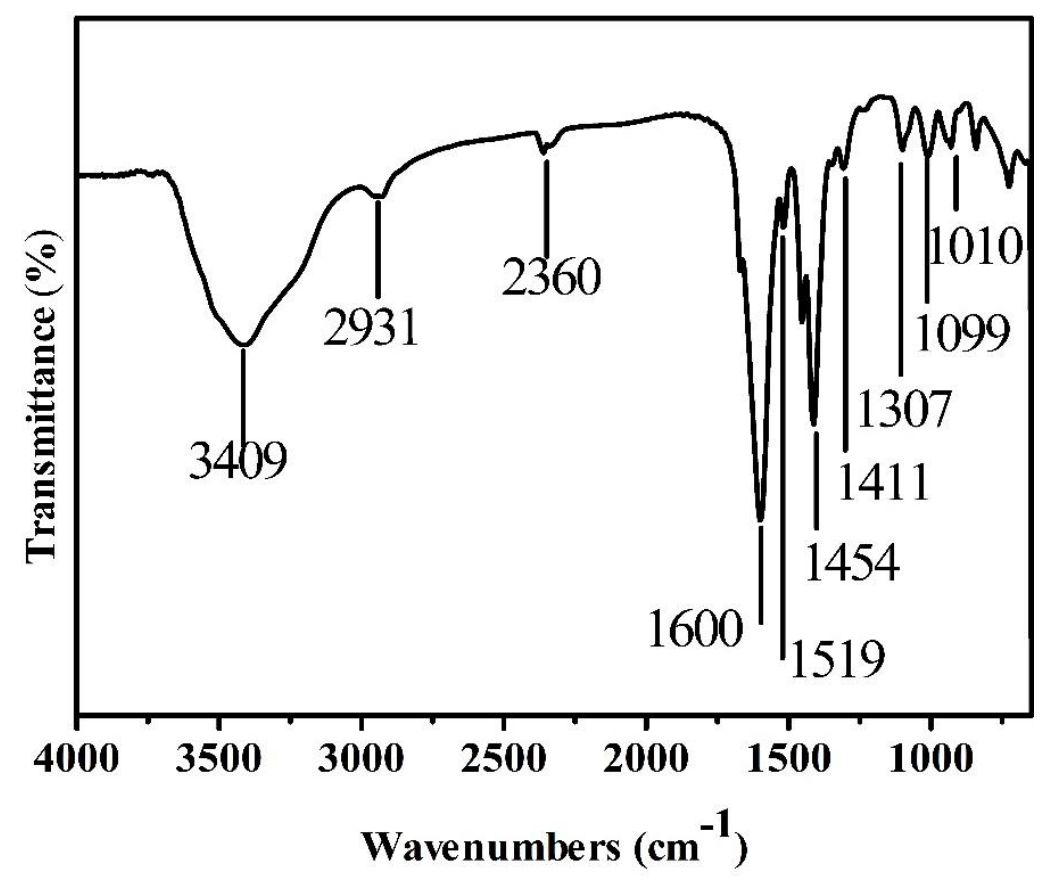

Figure S11. FT-IR spectra of compound 1.

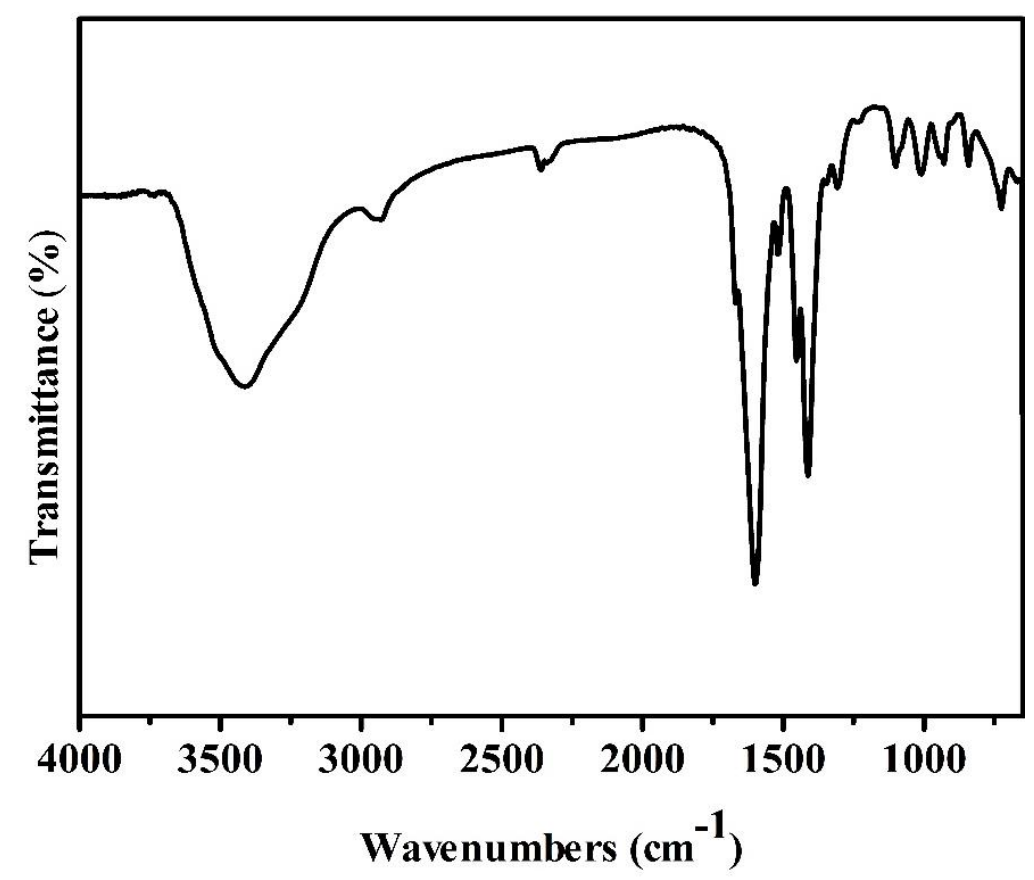

Figure S12. FT-IR spectra of compound 2. 
TG

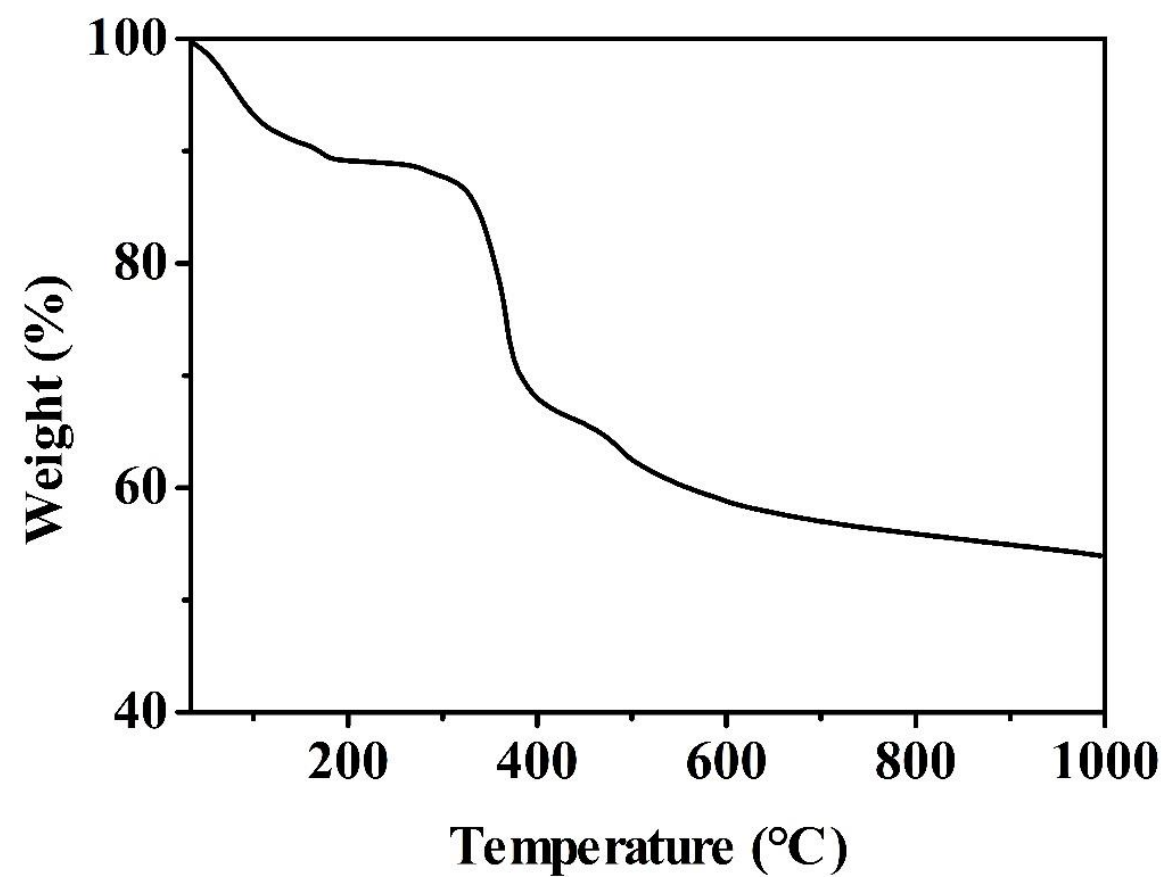

Figure S13. TG curves of compound 1.

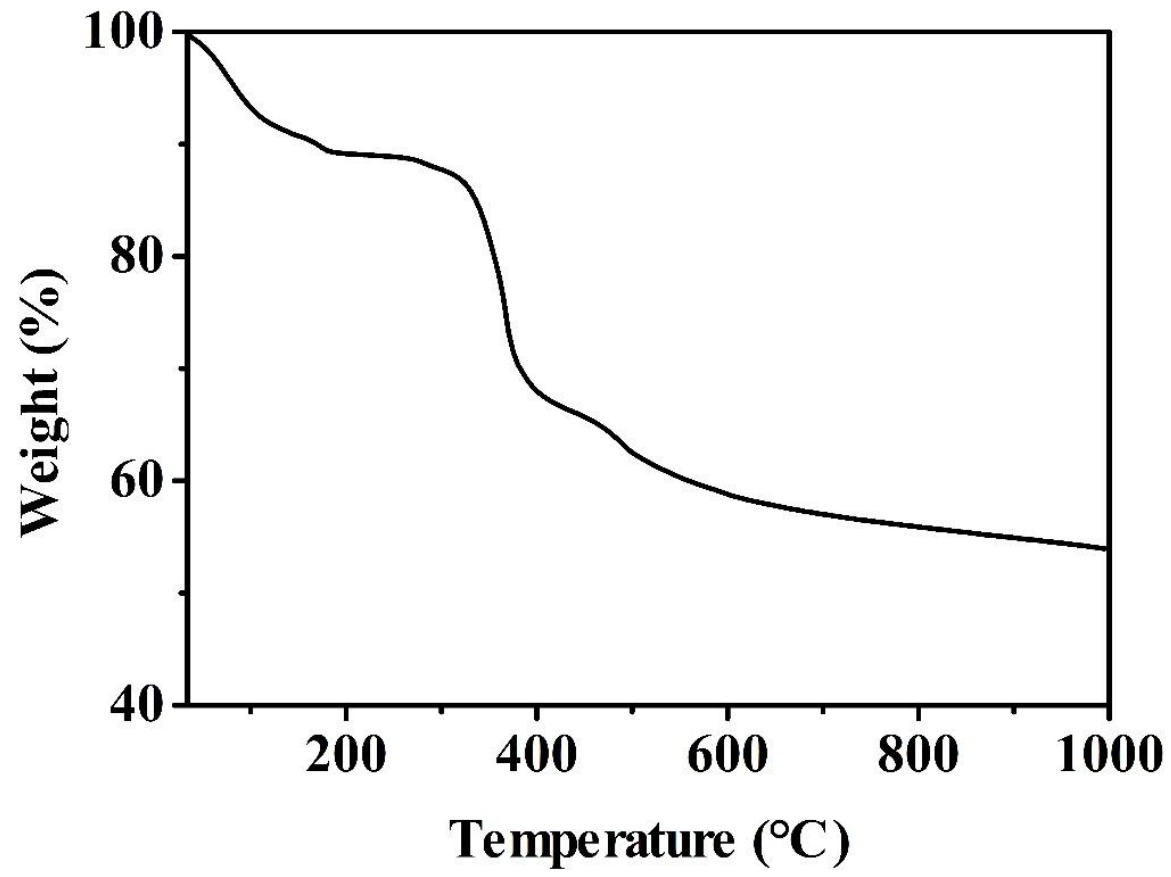

Figure S14. TG curves of compound 2. 
Magnetic properties.

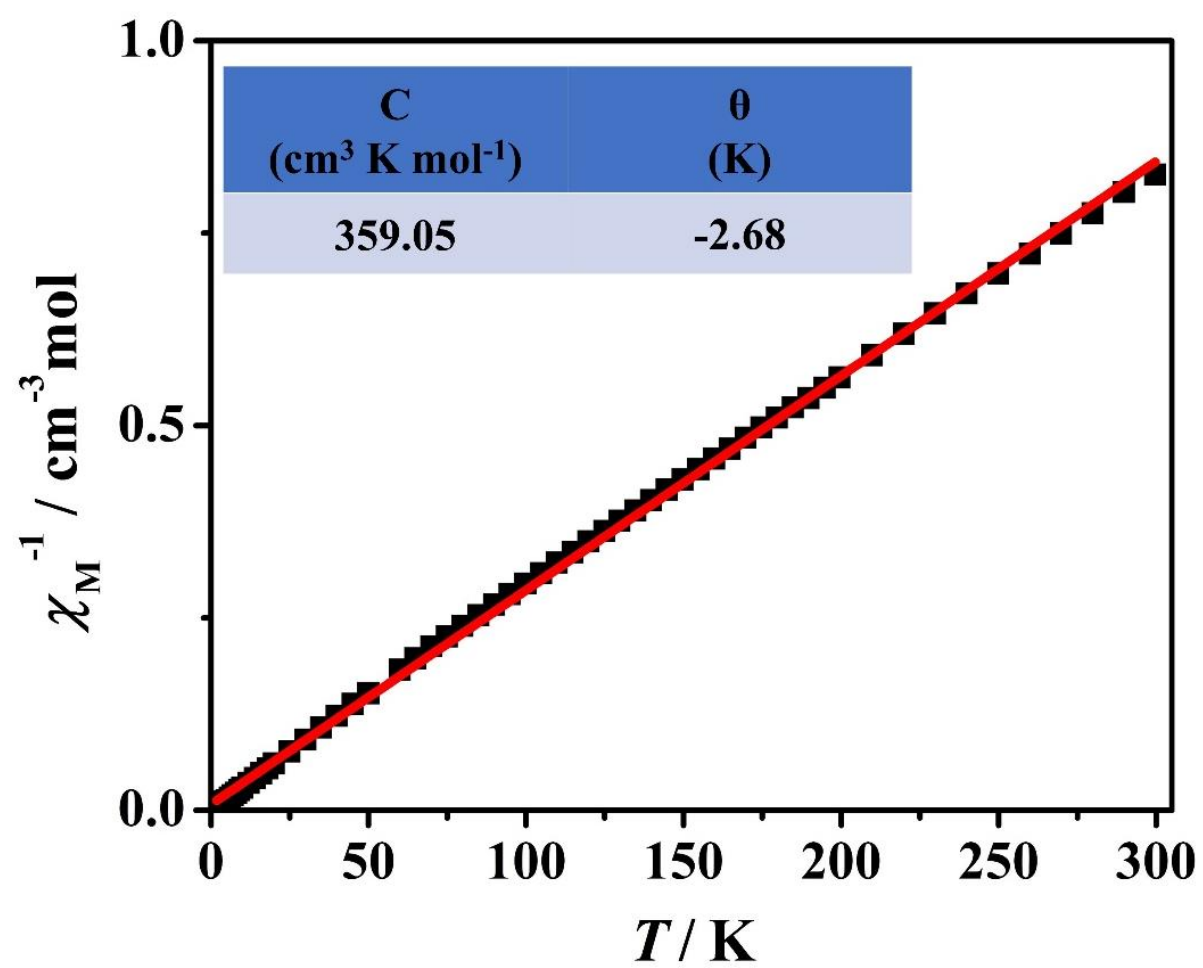

Figure S15. $\chi_{\mathrm{M}}{ }^{-1}$ versus $\mathrm{T}$ plot of $\mathbf{1}$. The red line is fitting result with $\chi_{\mathrm{M}}=\mathrm{C} /(\mathrm{T}-\theta)$.

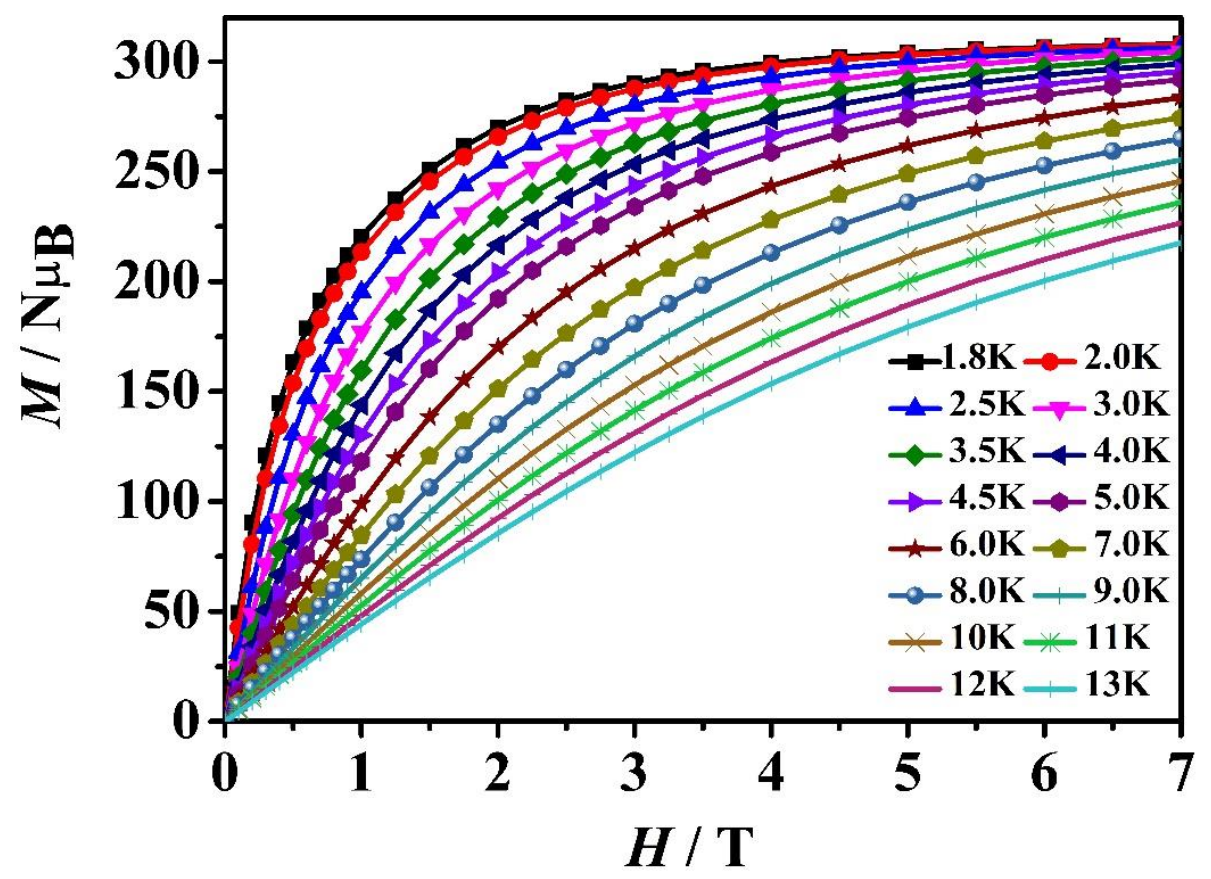

Figure S16. The isothermal field-dependence magnetization $(M-H)$ at low temperatures for $\mathbf{1}(1.8-13.0 \mathrm{~K})$. 


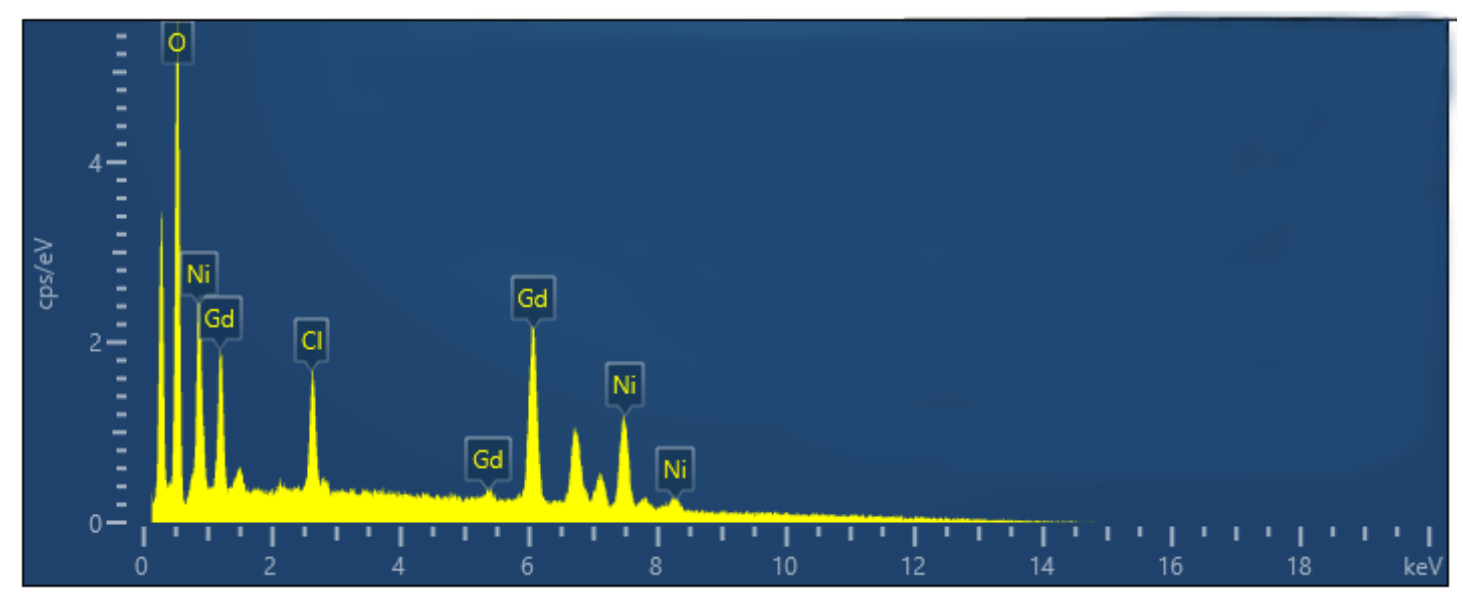

Figure S17. The EDS measurement of $\mathbf{1}$.

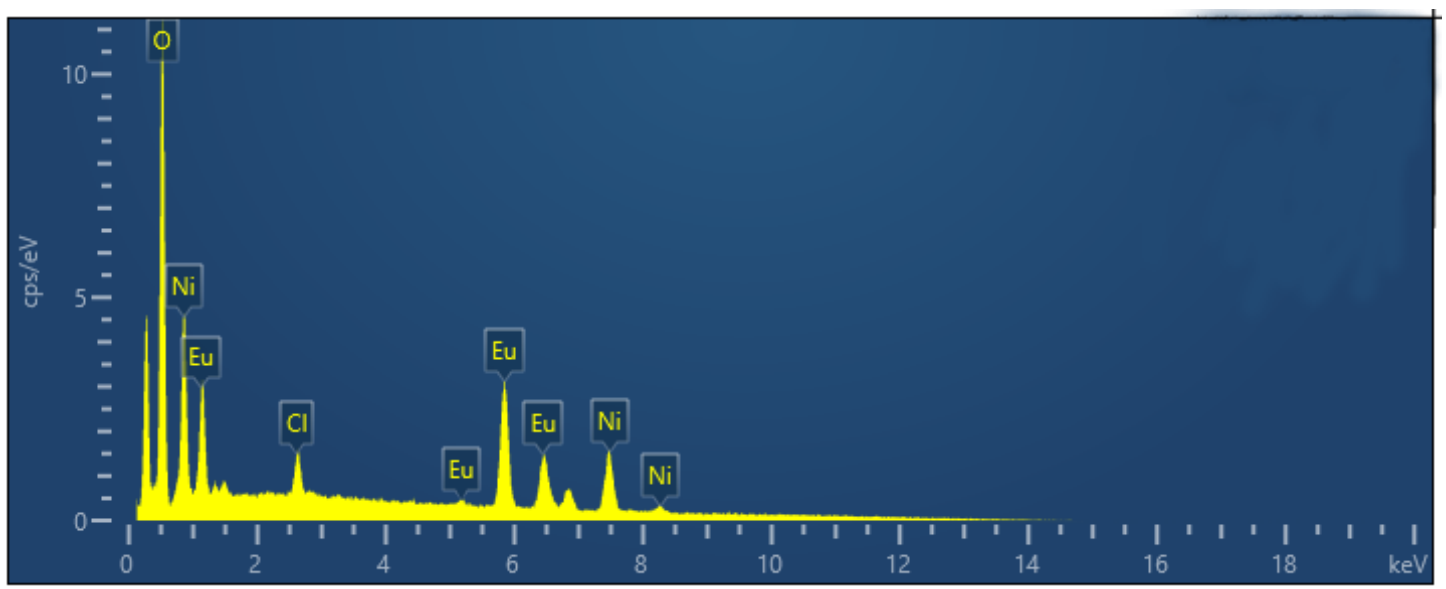

Figure S18. The EDS measurement of 2. 
Table S1. The selected bond lengths $(\AA)$ and bond angles $\left(^{\circ}\right)$ for compound 1.

\begin{tabular}{|c|c|c|c|}
\hline $\mathrm{Gd}(1)-\mathrm{O}(74)$ & $2.329(14)$ & $\mathrm{Ni}(1)-\mathrm{O}(30)$ & $2.038(13)$ \\
\hline $\mathrm{Gd}(1)-\mathrm{O}(22)$ & $2.433(13)$ & $\mathrm{Ni}(1)-\mathrm{O}(60)$ & $2.057(14)$ \\
\hline $\mathrm{Gd}(1)-\mathrm{O}(114)$ & $2.443(15)$ & $\mathrm{Ni}(1)-\mathrm{O}(78)$ & $2.057(14)$ \\
\hline $\mathrm{Gd}(1)-\mathrm{O}(53)$ & $2.465(13)$ & $\mathrm{Ni}(1)-\mathrm{O}(33)$ & $2.068(14)$ \\
\hline $\mathrm{Gd}(1)-\mathrm{O}(89)$ & $2.486(14)$ & $\mathrm{Ni}(1)-\mathrm{N}(13)$ & $2.080(17)$ \\
\hline $\mathrm{Gd}(1)-\mathrm{O}(18)$ & $2.498(13)$ & $\mathrm{Ni}(1)-\mathrm{O}(24)$ & $2.114(14)$ \\
\hline $\mathrm{Gd}(1)-\mathrm{O}(17)$ & $2.501(13)$ & $\mathrm{Ni}(2)-\mathrm{O}(86) \# 1$ & $2.028(15)$ \\
\hline $\mathrm{Gd}(1)-\mathrm{O}(36)$ & $2.513(14)$ & $\mathrm{Ni}(2)-\mathrm{N}(2)$ & $2.043(17)$ \\
\hline $\mathrm{Gd}(1)-\mathrm{O}(49)$ & $2.539(14)$ & $\mathrm{Ni}(2)-\mathrm{O}(109) \# 1$ & $2.052(13)$ \\
\hline $\mathrm{Gd}(2)-\mathrm{O}(21)$ & $2.400(13)$ & $\mathrm{Ni}(3)-\mathrm{O}(22)$ & $2.039(13)$ \\
\hline $\mathrm{Gd}(2)-\mathrm{O}(23)$ & $2.406(14)$ & $\mathrm{Ni}(3)-\mathrm{O}(65)$ & $2.053(14)$ \\
\hline $\mathrm{Gd}(2)-\mathrm{O}(93)$ & $2.417(14)$ & $\mathrm{Ni}(3)-\mathrm{O}(141)$ & $2.063(15)$ \\
\hline $\mathrm{Gd}(2)-\mathrm{O}(12)$ & $2.447(13)$ & $\mathrm{Ni}(3)-\mathrm{O}(26)$ & $2.064(14)$ \\
\hline $\mathrm{Gd}(2)-\mathrm{O}(56)$ & $2.454(12)$ & $\mathrm{Ni}(3)-\mathrm{O}(18)$ & $2.072(14)$ \\
\hline $\mathrm{Gd}(2)-\mathrm{O}(11)$ & $2.463(12)$ & $\mathrm{Ni}(3)-\mathrm{N}(14)$ & $2.073(18)$ \\
\hline $\mathrm{Gd}(2)-\mathrm{O}(67)$ & $2.483(13)$ & $\mathrm{Ni}(4)-\mathrm{O}(53)$ & $2.013(13)$ \\
\hline $\mathrm{Gd}(2)-\mathrm{O}(61)$ & $2.549(15)$ & $\mathrm{Ni}(4)-\mathrm{O}(28)$ & $2.032(14)$ \\
\hline $\mathrm{Gd}(2)-\mathrm{O}(5)$ & $2.587(13)$ & $\mathrm{Ni}(4)-\mathrm{O}(79)$ & $2.058(14)$ \\
\hline $\mathrm{Gd}(3)-\mathrm{O}(13)$ & $2.384(13)$ & $\mathrm{Ni}(4)-\mathrm{O}(135)$ & $2.067(14)$ \\
\hline $\mathrm{Gd}(3)-\mathrm{O}(15)$ & $2.424(13)$ & $\mathrm{Ni}(5)-\mathrm{O}(13)$ & $2.016(13)$ \\
\hline $\mathrm{Gd}(3)-\mathrm{O}(76)$ & $2.425(13)$ & $\mathrm{Ni}(5)-\mathrm{O}(144)$ & $2.041(15)$ \\
\hline $\mathrm{Gd}(3)-\mathrm{O}(59)$ & $2.428(14)$ & $\mathrm{Ni}(5)-\mathrm{O}(51)$ & $2.044(15)$ \\
\hline $\mathrm{Gd}(3)-\mathrm{O}(50)$ & $2.462(13)$ & $\mathrm{Ni}(5)-\mathrm{O}(119)$ & $2.067(14)$ \\
\hline $\mathrm{Gd}(3)-\mathrm{O}(30)$ & $2.464(13)$ & $\mathrm{Ni}(5)-\mathrm{O}(15)$ & $2.077(13)$ \\
\hline $\mathrm{Gd}(3)-\mathrm{O}(40)$ & $2.476(14)$ & $\mathrm{Ni}(5)-\mathrm{N}(5)$ & $2.088(17)$ \\
\hline $\mathrm{Gd}(3)-\mathrm{O}(7)$ & $2.491(13)$ & $\mathrm{Ni}(6)-\mathrm{O}(27)$ & $2.009(13)$ \\
\hline $\mathrm{Gd}(3)-\mathrm{O}(60)$ & $2.537(14)$ & $\mathrm{Ni}(6)-\mathrm{O}(136)$ & $2.026(16)$ \\
\hline $\mathrm{Gd}(4)-\mathrm{O}(57)$ & $2.330(14)$ & $\mathrm{Ni}(6)-\mathrm{O}(44)$ & $2.066(13)$ \\
\hline $\mathrm{Gd}(4)-\mathrm{O}(22)$ & $2.423(13)$ & $\mathrm{Ni}(6)-\mathrm{N}(12)$ & $2.073(17)$ \\
\hline $\mathrm{Gd}(4)-\mathrm{O}(76)$ & $2.449(13)$ & $\mathrm{Ni}(7)-\mathrm{O}(11)$ & $1.988(12)$ \\
\hline $\mathrm{Gd}(4)-\mathrm{O}(39)$ & $2.476(14)$ & $\mathrm{Ni}(7)-\mathrm{O}(134)$ & $2.013(16)$ \\
\hline $\mathrm{Gd}(4)-\mathrm{O}(65)$ & $2.477(14)$ & $\mathrm{Ni}(7)-\mathrm{N}(8)$ & $2.037(18)$ \\
\hline $\mathrm{Gd}(4)-\mathrm{O}(31)$ & $2.496(13)$ & $\mathrm{Ni}(7)-\mathrm{O}(63)$ & $2.058(14)$ \\
\hline $\mathrm{Gd}(4)-\mathrm{O}(17)$ & $2.518(13)$ & $\mathrm{Ni}(7)-\mathrm{O}(61)$ & $2.073(14)$ \\
\hline $\mathrm{Gd}(4)-\mathrm{O}(78)$ & $2.535(14)$ & $\mathrm{Ni}(7)-\mathrm{O}(52)$ & $2.088(14)$ \\
\hline $\mathrm{Gd}(4)-\mathrm{O}(30)$ & $2.542(13)$ & $\mathrm{Ni}(8)-\mathrm{O}(107)$ & $2.030(14)$ \\
\hline $\mathrm{O}(74)-\mathrm{Gd}(1)-\mathrm{O}(22)$ & $75.7(5)$ & $\mathrm{O}(21)-\mathrm{Gd}(2)-\mathrm{O}(23)$ & $119.3(5)$ \\
\hline $\mathrm{O}(74)-\mathrm{Gd}(1)-\mathrm{O}(114)$ & $85.8(5)$ & $\mathrm{O}(21)-\mathrm{Gd}(2)-\mathrm{O}(93)$ & $69.7(5)$ \\
\hline $\mathrm{O}(22)-\mathrm{Gd}(1)-\mathrm{O}(114)$ & $69.6(5)$ & $\mathrm{O}(23)-\mathrm{Gd}(2)-\mathrm{O}(93)$ & $131.6(5)$ \\
\hline
\end{tabular}




\begin{tabular}{|c|c|c|c|}
\hline $\mathrm{O}(74)-\mathrm{Gd}(1)-\mathrm{O}(53)$ & $134.3(5)$ & $\mathrm{O}(21)-\mathrm{Gd}(2)-\mathrm{O}(12)$ & $68.9(4)$ \\
\hline $\mathrm{O}(22)-\mathrm{Gd}(1)-\mathrm{O}(53)$ & $130.5(4)$ & $\mathrm{O}(23)-\mathrm{Gd}(2)-\mathrm{O}(12)$ & $155.3(4)$ \\
\hline $\mathrm{O}(114)-\mathrm{Gd}(1)-\mathrm{O}(53)$ & $134.9(5)$ & $\mathrm{O}(93)-\mathrm{Gd}(2)-\mathrm{O}(12)$ & $72.7(4)$ \\
\hline $\mathrm{O}(74)-\mathrm{Gd}(1)-\mathrm{O}(89)$ & $72.3(5)$ & $\mathrm{O}(21)-\mathrm{Gd}(2)-\mathrm{O}(56)$ & $132.2(4)$ \\
\hline $\mathrm{O}(22)-\mathrm{Gd}(1)-\mathrm{O}(89)$ & 131.2(4) & $\mathrm{O}(21)-\mathrm{Gd}(2)-\mathrm{O}(23)$ & $119.3(5)$ \\
\hline $\mathrm{O}(114)-\mathrm{Gd}(1)-\mathrm{O}(89)$ & $141.1(5)$ & $\mathrm{O}(21)-\mathrm{Gd}(2)-\mathrm{O}(93)$ & $69.7(5)$ \\
\hline $\mathrm{O}(53)-\mathrm{Gd}(1)-\mathrm{O}(89)$ & $62.6(4)$ & $\mathrm{O}(23)-\mathrm{Gd}(2)-\mathrm{O}(93)$ & $131.6(5)$ \\
\hline $\mathrm{O}(74)-\mathrm{Gd}(1)-\mathrm{O}(18)$ & $145.6(5)$ & $\mathrm{O}(21)-\mathrm{Gd}(2)-\mathrm{O}(12)$ & $68.9(4)$ \\
\hline $\mathrm{O}(22)-\mathrm{Gd}(1)-\mathrm{O}(18)$ & 69.9(4) & $\mathrm{O}(23)-\mathrm{Gd}(2)-\mathrm{O}(12)$ & $155.3(4)$ \\
\hline $\mathrm{O}(114)-\mathrm{Gd}(1)-\mathrm{O}(18)$ & $80.6(5)$ & $\mathrm{O}(93)-\mathrm{Gd}(2)-\mathrm{O}(12)$ & $72.7(4)$ \\
\hline $\mathrm{O}(53)-\mathrm{Gd}(1)-\mathrm{O}(18)$ & $73.7(4)$ & $\mathrm{O}(21)-\mathrm{Gd}(2)-\mathrm{O}(56)$ & $132.2(4)$ \\
\hline $\mathrm{O}(89)-\mathrm{Gd}(1)-\mathrm{O}(18)$ & $134.3(4)$ & $\mathrm{O}(23)-\mathrm{Gd}(2)-\mathrm{O}(56)$ & $75.8(4)$ \\
\hline $\mathrm{O}(74)-\mathrm{Gd}(1)-\mathrm{O}(17)$ & $86.1(5)$ & $\mathrm{O}(93)-\mathrm{Gd}(2)-\mathrm{O}(56)$ & $136.7(4)$ \\
\hline $\mathrm{O}(22)-\mathrm{Gd}(1)-\mathrm{O}(17)$ & 66.3(4) & $\mathrm{O}(12)-\mathrm{Gd}(2)-\mathrm{O}(56)$ & $82.1(4)$ \\
\hline $\mathrm{O}(114)-\mathrm{Gd}(1)-\mathrm{O}(17)$ & $135.9(5)$ & $\mathrm{O}(21)-\mathrm{Gd}(2)-\mathrm{O}(11)$ & $134.8(4)$ \\
\hline $\mathrm{O}(53)-\mathrm{Gd}(1)-\mathrm{O}(17)$ & $76.6(4)$ & $\mathrm{O}(23)-\mathrm{Gd}(2)-\mathrm{O}(11)$ & $105.3(4)$ \\
\hline $\mathrm{O}(89)-\mathrm{Gd}(1)-\mathrm{O}(17)$ & $75.5(4)$ & $\mathrm{O}(93)-\mathrm{Gd}(2)-\mathrm{O}(11)$ & $75.6(4)$ \\
\hline $\mathrm{O}(18)-\mathrm{Gd}(1)-\mathrm{O}(17)$ & $82.2(4)$ & $\mathrm{O}(12)-\mathrm{Gd}(2)-\mathrm{O}(11)$ & $73.7(4)$ \\
\hline $\mathrm{O}(74)-\mathrm{Gd}(1)-\mathrm{O}(36)$ & $134.5(5)$ & $\mathrm{O}(56)-\mathrm{Gd}(2)-\mathrm{O}(11)$ & $63.6(4)$ \\
\hline $\mathrm{O}(22)-\mathrm{Gd}(1)-\mathrm{O}(36)$ & $125.1(4)$ & $\mathrm{O}(21)-\mathrm{Gd}(2)-\mathrm{O}(67)$ & $68.4(4)$ \\
\hline $\mathrm{O}(114)-\mathrm{Gd}(1)-\mathrm{O}(36)$ & $69.5(5)$ & $\mathrm{O}(23)-\mathrm{Gd}(2)-\mathrm{O}(67)$ & $65.7(4)$ \\
\hline $\mathrm{O}(53)-\mathrm{Gd}(1)-\mathrm{O}(36)$ & $66.8(4)$ & $\mathrm{O}(93)-\mathrm{Gd}(2)-\mathrm{O}(67)$ & $77.5(4)$ \\
\hline $\mathrm{O}(89)-\mathrm{Gd}(1)-\mathrm{O}(36)$ & $103.6(4)$ & $\mathrm{O}(12)-\mathrm{Gd}(2)-\mathrm{O}(67)$ & $134.1(4)$ \\
\hline $\mathrm{O}(18)-\mathrm{Gd}(1)-\mathrm{O}(36)$ & $68.7(4)$ & $\mathrm{O}(56)-\mathrm{Gd}(2)-\mathrm{O}(67)$ & 141.1(4) \\
\hline $\mathrm{O}(17)-\mathrm{Gd}(1)-\mathrm{O}(36)$ & $138.0(4)$ & $\mathrm{O}(11)-\mathrm{Gd}(2)-\mathrm{O}(67)$ & $130.7(4)$ \\
\hline $\mathrm{O}(74)-\mathrm{Gd}(1)-\mathrm{O}(49)$ & $67.8(5)$ & $\mathrm{O}(21)-\mathrm{Gd}(2)-\mathrm{O}(61)$ & $125.4(4)$ \\
\hline $\mathrm{O}(22)-\mathrm{Gd}(1)-\mathrm{O}(49)$ & 126.1(4) & $\mathrm{O}(23)-\mathrm{Gd}(2)-\mathrm{O}(61)$ & $67.3(5)$ \\
\hline $\mathrm{O}(114)-\mathrm{Gd}(1)-\mathrm{O}(49)$ & $69.4(5)$ & $\mathrm{O}(93)-\mathrm{Gd}(2)-\mathrm{O}(61)$ & $70.3(5)$ \\
\hline $\mathrm{O}(53)-\mathrm{Gd}(1)-\mathrm{O}(49)$ & $103.3(4)$ & $\mathrm{O}(12)-\mathrm{Gd}(2)-\mathrm{O}(61)$ & $129.3(4)$ \\
\hline $\mathrm{O}(89)-\mathrm{Gd}(1)-\mathrm{O}(49)$ & $72.6(4)$ & $\mathrm{O}(56)-\mathrm{Gd}(2)-\mathrm{O}(61)$ & $102.4(4)$ \\
\hline $\mathrm{O}(18)-\mathrm{Gd}(1)-\mathrm{O}(49)$ & $133.5(4)$ & $\mathrm{O}(11)-\mathrm{Gd}(2)-\mathrm{O}(61)$ & $64.5(4)$ \\
\hline $\mathrm{O}(17)-\mathrm{Gd}(1)-\mathrm{O}(49)$ & $143.5(4)$ & $\mathrm{O}(67)-\mathrm{Gd}(2)-\mathrm{O}(61)$ & $67.8(4)$ \\
\hline $\mathrm{O}(36)-\mathrm{Gd}(1)-\mathrm{O}(49)$ & $67.9(4)$ & $\mathrm{O}(21)-\mathrm{Gd}(2)-\mathrm{O}(5)$ & $73.6(4)$ \\
\hline $\mathrm{O}(21)-\mathrm{Gd}(2)-\mathrm{O}(23)$ & $119.3(5)$ & $\mathrm{O}(23)-\mathrm{Gd}(2)-\mathrm{O}(5)$ & $67.3(4)$ \\
\hline
\end{tabular}


Table S2. The selected bond lengths $(\AA)$ and bond angles $\left(^{\circ}\right)$ for compound 2.

\begin{tabular}{|c|c|c|c|}
\hline $\mathrm{Eu}(1)-\mathrm{O}(59)$ & $2.400(11)$ & $\mathrm{Eu}(5)-\mathrm{O}(32)$ & $2.397(10)$ \\
\hline $\mathrm{Eu}(1)-\mathrm{O}(128)$ & $2.414(11)$ & $\mathrm{Eu}(5)-\mathrm{O}(20)$ & $2.419(10)$ \\
\hline $\mathrm{Eu}(1)-\mathrm{O}(11)$ & $2.436(10)$ & $\mathrm{Eu}(5)-\mathrm{O}(41) \# 1$ & $2.467(11)$ \\
\hline $\mathrm{Eu}(1)-\mathrm{O}(23)$ & $2.470(10)$ & $\mathrm{Eu}(5)-\mathrm{O}(25)$ & $2.494(10)$ \\
\hline $\mathrm{Eu}(1)-\mathrm{O}(124)$ & $2.473(11)$ & $\mathrm{Eu}(5)-\mathrm{O}(21)$ & $2.506(10)$ \\
\hline $\mathrm{Eu}(1)-\mathrm{O}(78)$ & $2.510(11)$ & $\mathrm{Eu}(5)-\mathrm{O}(5)$ & $2.525(10)$ \\
\hline $\mathrm{Eu}(1)-\mathrm{O}(82)$ & $2.514(10)$ & $\mathrm{Eu}(5)-\mathrm{O}(66)$ & $2.533(11)$ \\
\hline $\mathrm{Eu}(1)-\mathrm{O}(63)$ & $2.578(11)$ & $\mathrm{Eu}(5)-\mathrm{O}(90)$ & $2.586(12)$ \\
\hline $\mathrm{Eu}(1)-\mathrm{O}(19)$ & $2.620(10)$ & $\mathrm{Eu}(6)-\mathrm{O}(43)$ & $2.315(10)$ \\
\hline $\mathrm{Eu}(2)-\mathrm{O}(83)$ & $2.345(11)$ & $\mathrm{Eu}(6)-\mathrm{O}(92)$ & $2.380(11)$ \\
\hline $\mathrm{Eu}(2)-\mathrm{O}(88)$ & $2.427(11)$ & $\mathrm{Eu}(6)-\mathrm{O}(138)$ & $2.382(12)$ \\
\hline $\mathrm{Eu}(2)-\mathrm{O}(102)$ & $2.458(11)$ & $\mathrm{Eu}(6)-\mathrm{O}(73)$ & $2.400(11)$ \\
\hline $\mathrm{Eu}(2)-\mathrm{O}(58)$ & $2.463(10)$ & $\mathrm{Eu}(6)-\mathrm{O}(95)$ & $2.426(12)$ \\
\hline $\mathrm{Eu}(2)-\mathrm{O}(96)$ & $2.485(12)$ & $\mathrm{Eu}(6)-\mathrm{O}(15)$ & $2.494(10)$ \\
\hline $\mathrm{Eu}(2)-\mathrm{O}(50)$ & $2.493(11)$ & $\mathrm{Eu}(6)-\mathrm{O}(35)$ & $2.552(10)$ \\
\hline $\mathrm{Eu}(2)-\mathrm{O}(26)$ & $2.516(11)$ & $\mathrm{Eu}(6)-\mathrm{O}(109)$ & $2.727(11)$ \\
\hline $\mathrm{Eu}(2)-\mathrm{O}(38)$ & $2.535(10)$ & $\mathrm{Eu}(6)-\mathrm{O}(150)$ & $2.738(13)$ \\
\hline $\mathrm{Eu}(2)-\mathrm{O}(37)$ & $2.550(11)$ & $\mathrm{Eu}(6)-\mathrm{C}(1 \mathrm{~A})$ & $2.910(15)$ \\
\hline $\mathrm{Eu}(3)-\mathrm{O}(29)$ & $2.386(10)$ & $\mathrm{Eu}(7)-\mathrm{O}(106)$ & $2.394(10)$ \\
\hline $\mathrm{Eu}(3)-\mathrm{O}(33)$ & $2.422(10)$ & $\mathrm{Eu}(7)-\mathrm{O}(69)$ & $2.417(11)$ \\
\hline $\mathrm{Eu}(3)-\mathrm{O}(69)$ & $2.434(11)$ & $\mathrm{Eu}(7)-\mathrm{O}(92)$ & $2.435(11)$ \\
\hline $\mathrm{Eu}(3)-\mathrm{O}(93)$ & $2.453(10)$ & $\mathrm{Eu}(7)-\mathrm{O}(17)$ & $2.441(10)$ \\
\hline $\mathrm{Eu}(3)-\mathrm{O}(51)$ & $2.472(10)$ & $\mathrm{Eu}(7)-\mathrm{O}(125)$ & $2.446(11)$ \\
\hline $\mathrm{Eu}(3)-\mathrm{O}(42)$ & $2.480(11)$ & $\mathrm{Eu}(7)-\mathrm{O}(67)$ & $2.462(12)$ \\
\hline $\mathrm{Eu}(3)-\mathrm{O}(16)$ & $2.498(10)$ & $\mathrm{Eu}(7)-\mathrm{O}(35)$ & $2.487(10)$ \\
\hline $\mathrm{Eu}(3)-\mathrm{O}(8)$ & $2.519(10)$ & $\mathrm{Eu}(7)-\mathrm{O}(97)$ & $2.554(12)$ \\
\hline $\mathrm{Eu}(3)-\mathrm{O}(57)$ & $2.554(12)$ & $\mathrm{Eu}(7)-\mathrm{O}(51)$ & $2.670(9)$ \\
\hline $\mathrm{Eu}(4)-\mathrm{O}(32)$ & $2.368(10)$ & $\mathrm{Eu}(8)-\mathrm{O}(65)$ & $2.363(11)$ \\
\hline $\mathrm{Eu}(4)-\mathrm{O}(36)$ & $2.435(10)$ & $\mathrm{Eu}(8)-\mathrm{O}(88)$ & $2.449(11)$ \\
\hline $\mathrm{Eu}(4)-\mathrm{O}(82)$ & $2.447(10)$ & $\mathrm{Eu}(8)-\mathrm{O}(93)$ & $2.453(10)$ \\
\hline $\mathrm{Eu}(4)-\mathrm{O}(23)$ & $2.453(10)$ & $\mathrm{Eu}(8)-\mathrm{O}(34)$ & $2.456(11)$ \\
\hline $\mathrm{Eu}(4)-\mathrm{O}(21)$ & $2.458(10)$ & $\mathrm{Eu}(8)-\mathrm{O}(60)$ & $2.500(11)$ \\
\hline $\mathrm{Eu}(4)-\mathrm{O}(81)$ & $2.464(11)$ & $\mathrm{Eu}(8)-\mathrm{O}(27)$ & $2.502(11)$ \\
\hline $\mathrm{Eu}(4)-\mathrm{O}(68)$ & $2.516(11)$ & $\mathrm{Eu}(8)-\mathrm{O}(26)$ & $2.533(11)$ \\
\hline $\mathrm{Eu}(4)-\mathrm{O}(54)$ & $2.537(10)$ & $\mathrm{Eu}(8)-\mathrm{O}(16)$ & $2.550(9)$ \\
\hline $\mathrm{Eu}(4)-\mathrm{O}(30)$ & $2.630(10)$ & $\mathrm{Eu}(8)-\mathrm{O}(132)$ & $2.548(12)$ \\
\hline $\mathrm{Eu}(5)-\mathrm{O}(12)$ & $2.397(10)$ & $\mathrm{Eu}(9)-\mathrm{O}(20)$ & $2.402(10)$ \\
\hline $\mathrm{O}(59)-\mathrm{Eu}(1)-\mathrm{O}(128)$ & 70.1(4) & $\mathrm{O}(82)-\mathrm{Eu}(1)-\mathrm{O}(63)$ & $102.2(3)$ \\
\hline $\mathrm{O}(59)-\mathrm{Eu}(1)-\mathrm{O}(11)$ & 118.3(4) & $\mathrm{O}(59)-\mathrm{Eu}(1)-\mathrm{O}(19)$ & $73.3(3)$ \\
\hline $\mathrm{O}(128)-\mathrm{Eu}(1)-\mathrm{O}(11)$ & $131.3(3)$ & $\mathrm{O}(128)-\mathrm{Eu}(1)-\mathrm{O}(19)$ & $143.4(4)$ \\
\hline
\end{tabular}




\begin{tabular}{|c|c|c|c|}
\hline $\mathrm{O}(59)-\mathrm{Eu}(1)-\mathrm{O}(23)$ & 136.2(4) & $\mathrm{O}(11)-\mathrm{Eu}(1)-\mathrm{O}(19)$ & $67.6(3)$ \\
\hline $\mathrm{O}(128)-\mathrm{Eu}(1)-\mathrm{O}(23)$ & $76.6(4)$ & $\mathrm{O}(23)-\mathrm{Eu}(1)-\mathrm{O}(19)$ & $134.4(3)$ \\
\hline $\mathrm{O}(11)-\mathrm{Eu}(1)-\mathrm{O}(23)$ & $105.0(3)$ & $\mathrm{O}(124)-\mathrm{Eu}(1)-\mathrm{O}(19)$ & $94.6(3)$ \\
\hline $\mathrm{O}(59)-\mathrm{Eu}(1)-\mathrm{O}(124)$ & $69.0(4)$ & $\mathrm{O}(78)-\mathrm{Eu}(1)-\mathrm{O}(19)$ & $89.2(3)$ \\
\hline $\mathrm{O}(128)-\mathrm{Eu}(1)-\mathrm{O}(124)$ & $73.2(4)$ & $\mathrm{O}(82)-\mathrm{Eu}(1)-\mathrm{O}(19)$ & $72.5(3)$ \\
\hline $\mathrm{O}(11)-\mathrm{Eu}(1)-\mathrm{O}(124)$ & $155.3(4)$ & $\mathrm{O}(63)-\mathrm{Eu}(1)-\mathrm{O}(19)$ & $135.3(3)$ \\
\hline $\mathrm{O}(23)-\mathrm{Eu}(1)-\mathrm{O}(124)$ & $74.7(4)$ & $\mathrm{O}(83)-\mathrm{Eu}(2)-\mathrm{O}(88)$ & $76.7(4)$ \\
\hline $\mathrm{O}(59)-\mathrm{Eu}(1)-\mathrm{O}(78)$ & 69.1(4) & $\mathrm{O}(83)-\mathrm{Eu}(2)-\mathrm{O}(102)$ & $86.8(4)$ \\
\hline $\mathrm{O}(128)-\mathrm{Eu}(1)-\mathrm{O}(78)$ & $77.3(4)$ & $\mathrm{O}(88)-\mathrm{Eu}(2)-\mathrm{O}(102)$ & $69.6(4)$ \\
\hline $\mathrm{O}(11)-\mathrm{Eu}(1)-\mathrm{O}(78)$ & $64.7(3)$ & $\mathrm{O}(83)-\mathrm{Eu}(2)-\mathrm{O}(58)$ & $71.2(4)$ \\
\hline $\mathrm{O}(23)-\mathrm{Eu}(1)-\mathrm{O}(78)$ & $129.9(3)$ & $\mathrm{O}(88)-\mathrm{Eu}(2)-\mathrm{O}(58)$ & $130.6(4)$ \\
\hline $\mathrm{O}(124)-\mathrm{Eu}(1)-\mathrm{O}(78)$ & $134.8(4)$ & $\mathrm{O}(102)-\mathrm{Eu}(2)-\mathrm{O}(58)$ & $141.8(4)$ \\
\hline $\mathrm{O}(59)-\mathrm{Eu}(1)-\mathrm{O}(82)$ & $132.4(3)$ & $\mathrm{O}(83)-\mathrm{Eu}(2)-\mathrm{O}(96)$ & $135.6(4)$ \\
\hline $\mathrm{O}(128)-\mathrm{Eu}(1)-\mathrm{O}(82)$ & $136.2(4)$ & $\mathrm{O}(88)-\mathrm{Eu}(2)-\mathrm{O}(96)$ & $123.7(4)$ \\
\hline $\mathrm{O}(11)-\mathrm{Eu}(1)-\mathrm{O}(82)$ & $76.6(3)$ & $\mathrm{O}(102)-\mathrm{Eu}(2)-\mathrm{O}(96)$ & $69.0(4)$ \\
\hline $\mathrm{O}(23)-\mathrm{Eu}(1)-\mathrm{O}(82)$ & $62.2(3)$ & $\mathrm{O}(58)-\mathrm{Eu}(2)-\mathrm{O}(96)$ & $105.4(4)$ \\
\hline $\mathrm{O}(124)-\mathrm{Eu}(1)-\mathrm{O}(82)$ & $81.8(3)$ & $\mathrm{O}(83)-\mathrm{Eu}(2)-\mathrm{O}(50)$ & $145.6(4)$ \\
\hline $\mathrm{O}(78)-\mathrm{Eu}(1)-\mathrm{O}(82)$ & $141.2(3)$ & $\mathrm{O}(88)-\mathrm{Eu}(2)-\mathrm{O}(50)$ & $69.0(4)$ \\
\hline $\mathrm{O}(59)-\mathrm{Eu}(1)-\mathrm{O}(63)$ & $125.5(4)$ & $\mathrm{O}(102)-\mathrm{Eu}(2)-\mathrm{O}(50)$ & $79.4(4)$ \\
\hline $\mathrm{O}(128)-\mathrm{Eu}(1)-\mathrm{O}(63)$ & $69.9(4)$ & $\mathrm{O}(58)-\mathrm{Eu}(2)-\mathrm{O}(50)$ & $135.0(3)$ \\
\hline $\mathrm{O}(11)-\mathrm{Eu}(1)-\mathrm{O}(63)$ & $68.0(3)$ & $\mathrm{O}(96)-\mathrm{Eu}(2)-\mathrm{O}(50)$ & $67.3(4)$ \\
\hline $\mathrm{O}(23)-\mathrm{Eu}(1)-\mathrm{O}(63)$ & $63.9(3)$ & $\mathrm{O}(83)-\mathrm{Eu}(2)-\mathrm{O}(26)$ & $86.1(4)$ \\
\hline $\mathrm{O}(124)-\mathrm{Eu}(1)-\mathrm{O}(63)$ & $129.3(4)$ & $\mathrm{O}(88)-\mathrm{Eu}(2)-\mathrm{O}(26)$ & $66.7(3)$ \\
\hline $\mathrm{O}(78)-\mathrm{Eu}(1)-\mathrm{O}(63)$ & $67.2(4)$ & $\mathrm{O}(102)-\mathrm{Eu}(2)-\mathrm{O}(26)$ & $136.2(4)$ \\
\hline
\end{tabular}

